EPJ Web of Conferences 66, 04019 (2014)

DOI: $10.1051 /$ epjconf/ 20146604019

(C) Owned by the authors, published by EDP Sciences, 2014

\title{
Small-x Physics in eA at the LHeC: Understanding the Initial State of URHIC
}

\author{
Néstor Armesto ${ }^{1,2, a}$ for the LHeC Study Group \\ ${ }^{1}$ Departamento de Física de Partículas and IGFAE, Universidade de Santiago de Compostela, \\ 15706 Santiago de Compostela, Galicia-Spain \\ ${ }^{2}$ Physics Department, Theory Unit, CERN, CH-1211 Genève 23, Switzerland
}

\begin{abstract}
I discuss the project of an electron-proton/ion collider at CERN using the LHC beams, the Large Hadron Electron Collider. After a brief introduction, I summarise the possibilities for electron-nucleus collisions. I also indicate the status of the project and future steps to be taken.
\end{abstract}

\section{Introduction}

The parton structure of nuclei is largely unknown, main due to the lack of experimental data in most of the kinematical regions in parton momentum fraction $x$ and inverse resolution squared $Q^{2}$. This lack of knowledge reflects directly on predictions for high-energy heavy-ion collisions that constitute the basis of standard, conventional physics on top of which the effects of new phases of QCD matter should be observed. Therefore, our ability for a precise characterisation of the medium produced in ultrarelativistic heavy-ion collisions becomes limited by the lack of control on the conventional physics to be expected in such collisions.

On the other hand, the region of small $x$ is of fundamental interest because there the behaviour of QCD at large energies should be reflected. This behaviour is currently an open problem for the proton: presently existing experimental data at small- $x$ can be described by non-perturbative models and, more interestingly, by different realisations of evolution equations within perturbative QCD: linear (either fixed-order perturbation theory or resummation schemes) and non-linear approaches. Such non-linear phenomena are unavoidable from a theoretical point of view and saturation of partonic densities is expected to occur at high energies or small Bjorken- $x$. A specific realisation of QCD, the Color Glass Condensate, offers a non-perturbative but weak coupling realisation of saturation ideas, see [1] and references therein. The present discussion lies on the relevant kinematical regime for such phenomena and on the possibilities offered by experimental data to unravel their existence.

Prominently, the study of electron-ion collisions offers the possibility to clarify many aspect of direct relevance for heavy-ion collisions. The initial stage of the collision is determined by the wave function of the incoming nuclei, to be explored at an electron-ion collider. There you can also investigate the mechanism of particle production on nuclear targets and the dynamics that lead to a nearly

a e-mail: nestor.armesto@usc.es

This is an Open Access article distributed under the terms of the Creative Commons Attribution License 2.0, which permits unrestricted use, distribution, and reproduction in any medium, provided the original work is properly cited. 
isotropised system that subsequently behaves in a way that can be described by relativistic hydrodynamics. Finally, some of the techniques used to analyse the produced medium rely on changes in QCD radiation and hadronisation that can be addressed in a simpler setup in electron-ion collisions.

The Large Hadron-electron Collider (LHeC, see the Conceptual Design Report in [2] and contributions to the European Strategy Update in [3]) is an electron-proton/ion collider currently under design at CERN, which will collide $20 \div 140 \mathrm{GeV} e^{ \pm}$against the LHC beams, with a nominal luminosity of $10^{33} \mathrm{~cm}^{-2} \mathrm{~s}^{-1}$, although more recent studies indicate that $10^{34} \mathrm{~cm}^{-2} \mathrm{~s}^{-1}$ and integrated luminosities of order $1 \mathrm{ab}^{-1}$ are within reach (see [4]). The machine is subject, apart from several requirements on detector performance and power consumption, to the constraints of creating minimal disturbance during construction and working simultaneously to the LHC. Besides Higgs and electro-weak studies and searches for new physics, this machine will perform precision QCD studies and it should allow unambiguous access to the novel regime of QCD in which unitarity constraints are at work - the dense region shown in figure 1 . As the transition between the dilute and the dense regions is a density effect, a two-pronged approach must be pursued: decreasing $x$ at fixed mass number $A$ and $Q^{2}$, and also increasing $A$ at fixed $x$ and $Q^{2}$. The LHeC will give access to a completely new region of the $Q^{2}-x$ plane for protons and, more prominently, for nuclei, see figure 2.
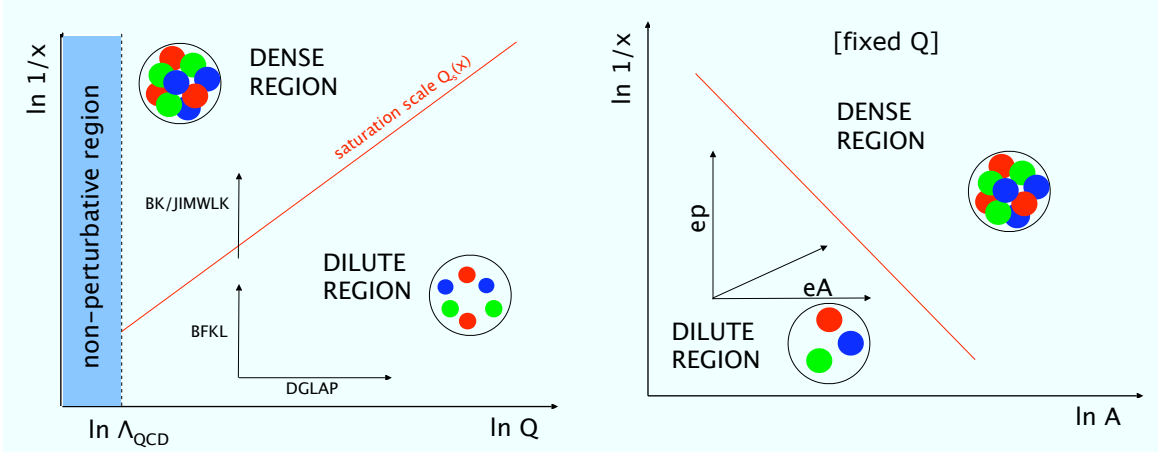

Figure 1. Sketch of the access to the dense partonic region where unitarity effects are essential, from the dilute one where linear evolution is valid. See the text for explanations. (From [2].)

In this contribution I will mention some aspects of the nuclear studies that may be performed at the LHeC. More information can be found in $[2,3,5]$. With the proposal included in the 2010 LRP of NuPECC, and CERN mandate to proceed towards a technical design in 3-4 years, future steps to be taken are the design of the detector and of the energy-recovery accelerator, and in the study of its complementarity with the LHC.

\section{Inclusive observables at small $x$}

The $\mathrm{LHeC}$ will give access to a completely new region of the $Q^{2}-x$ plane, see figure 2 . With this huge kinematical lever arm and the possibility to measure not only the total structure function, $F_{2}$, but also its flavor decomposition and the longitudinal one, $F_{L}$, (see figure 3 for examples of LHeC pseudodata on nuclear ratios of $F_{2}$ and $F_{L}$ ), the LHeC offers huge possibilities for constraining the parton density functions (PDFs) in DGLAP analysis in $e$ A collisions (see [2]), particularly for sea quarks and gluons, including the largely unconstrained flavour decomposition. As shown in figure 3 for $F_{2}$ and $F_{L}$, the 
expected uncertainty of $F_{2}$ data is much smaller than the spread of existing models. More information can be found in [5].

Note that the $\mathrm{LHeC}$ will explore a region of the $x$ - $Q^{2}$ plane overlapping with that accessible in $p \mathrm{~Pb}$ and $\mathrm{PbPb}$ collisions at the LHC but in a cleaner experimental setup and on firmer theoretical grounds, see [2].

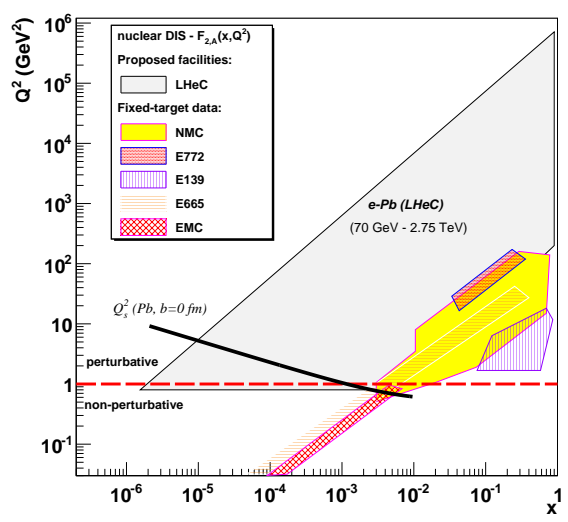

Figure 2. Region of the $Q^{2}-x$ plane that will be explored with the $\mathrm{LHeC}$ in $e \mathrm{~Pb}$, compared to those achievable at existing $e$ A experiments. An estimation of the saturation scale indicating the dilute-dense transition is shown (the black thick solid line). (From [2].)
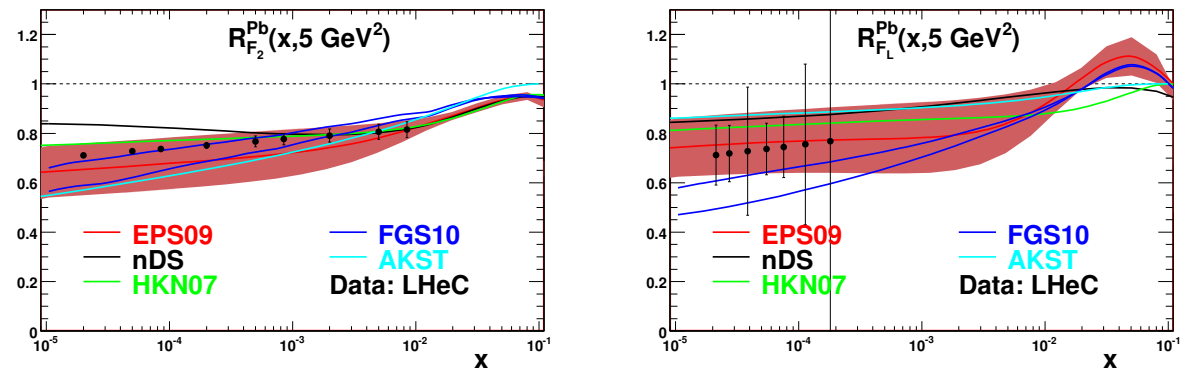

Figure 3. Predictions from different models for the nuclear ratio $R_{F_{i}}^{\mathrm{Pb}}=F_{i}^{\mathrm{Pb}} /\left(208 F_{i}^{\mathrm{p}}\right), i=2, L$, at small $x$, see the legend on the plots. Filled circles are LHeC pseudodata. (From [2].)

\section{Diffractive observables}

On diffraction $^{1}$ [2], the $\mathrm{LHeC}$ will explore a new domain of very low $\beta$ (e.g. down to a few $10^{-4}$ for $Q^{2} \sim 4 \mathrm{GeV}^{2}$ at $x_{P}=0.003$, two orders of magnitude smaller than at HERA, for $p$; for nuclei, information on diffraction at small $x$ does not exist). Several aspects can be highlighted:

\footnotetext{
${ }^{1}$ The diffractive kinematical variables are $x_{P}=\left(M_{X}^{2}-t+Q^{2}\right) /\left(W^{2}+Q^{2}\right)$ and $\beta=x / x_{P}$, with $M_{X}$ the diffractive mass, $t$ the transverse momentum squared and $W$ the $\gamma^{*}$-hadron center-of-mass energy.
} 
- It will give access to $M_{X}$ as large as $200 \mathrm{GeV}$, providing data to check models describing the transition from low to high $M_{X}$, and to constrain nuclear diffractive PDFs in DGLAP analyses.

- For elastic vector meson production or deeply virtual Compton scattering, a huge lever arm in $W$ will be explored (e.g. up to $\approx 1.2 \mathrm{TeV}$ for $e p$ with $E_{e}=50 \mathrm{GeV}$ ) with enough precision to disentangle linear evolution schemes from non-linear ones. Besides, the differential spectrum in $t$ will be accessible down to $t \sim-2 \mathrm{GeV}^{2}$. All this will also constrain quark and gluon densities and their transverse profile in hadrons and nuclei, at small $x$ where they are presently unknown.

- Gribov relation between diffraction in $e p$ and nuclear shadowing will be checked in a single experimental setup (see e.g. the FGS10 and AKST models in figure 3, the others being NLO DGLAP analyses).

- Stringent tests of factorisation in nuclei will be done through the measurements of diffractive and non-diffractive dijets .

The experimental challenge in separating inclusive diffraction $\left(e+\mathrm{A} \rightarrow e+X+X^{\prime}\right.$ with a rapidity gap) from coherent $(e+\mathrm{A} \rightarrow e+X+\mathrm{A})$ and incoherent $(e+\mathrm{A} \rightarrow e+X+Z \mathrm{p}+[A-Z] \mathrm{n})$ is under study.

\section{Final states}

The $\mathrm{LHeC}$ will offer huge possibilities for clarifying the dynamics of QCD radiation and hadronization. For example:

- The dynamics of QCD radiation at small $x$ will be analysed through forward jet and particle production, which will be abundant. As an example, the disappearance of back-back-correlations at small $x$, analogous to the one studied in dAu at RHIC in the forward region.

- The parton/hadron energy loss mechanism in semi-inclusive DIS will be tested by introducing a piece of coloured material - the nucleus - which would modify the hadronisation pattern i.e. its dependence on the traversed length (by varying either the impact parameter or the nuclear size), its chemical composition,... Energies as high as $10^{5} \mathrm{GeV}$ in the rest frame of the nucleus will be accessible and the transition from moderate to high energies, complementary to the low energies accessible in fixed-target experiments, will be studied.

As an example of the abundant yield of high-energy probes, inclusive jet rates for $Q^{2}=0$ around $10^{3}$ jets per $\mathrm{GeV}$ per year are expected [2] with $E_{T \text { jet }} \sim 95(80) \mathrm{GeV}$ in ep $(e \mathrm{~Pb})$.

In conclusion, I thank the organizers for the invitation to such a nice meeting. I gratefully acknowledge financial support by the ERC grant HotLHC ERC-2001-StG-279579; by Ministerio de Ciencia e Innovación of Spain grants FPA2009-06867-E, FPA2011-22776 and Consolider-Ingenio 2010 CPAN CSD2007-00042; by Xunta de Galicia grant PGIDIT10PXIB 206017PR; and by FEDER.

\section{References}

[1] F. Gelis, E. Iancu, J. Jalilian-Marian and R. Venugopalan, Ann. Rev. Nucl. Part. Sci. 60 (2010) 463 [arXiv:1002.0333 [hep-ph]].

[2] J. L. Abelleira Fernandez et al. [LHeC Study Group Collaboration], J. Phys. G 39 (2012) 075001 [arXiv:1206.2913 [physics.acc-ph]]; http://cern.ch/lhec.

[3] J. L. Abelleira Fernandez et al. [LHeC Study Group Collaboration], arXiv:1211.4831 [hep-ex]; arXiv:1211.5102 [hep-ex].

[4] O. Brüning and M. Klein, Mod. Phys. Lett. A 28 (2013) 16, 1330011 [arXiv:1305.2090 [physics.acc-ph]].

[5] H. Paukkunen et al. [for the LHeC Study Collaboration], arXiv:1306.2486 [hep-ph]. 